\title{
Comparison of secure messaging application (WhatsApp) and standard telephone usage for consultations on Length of Stay in the ED
}

\section{A prospective randomized controlled study}

Umut Gulacti; Ugur Lok

Adiyaman University, Adiyaman, Turkey

\section{Keywords}

Consultation, WhatsApp, secure messaging application, length of stay, overcrowding, emergency department

\section{Summary}

Objective: Consultation, the process of an Emergency Physician seeking an opinion from other specialties, occurs frequently in the Emergency Department (ED). The aim of this study was to determine the effect of secure messaging application (WhatsApp) usage for medical consultations on Emergency Department Length of Stay (ED LOS) and consult time. Methods: We conducted a prospective, randomized controlled trial in the ED using allocation concealment over three months. Consultations requested in the ED were allocated into two groups: consultations requested via the secure messaging application and consultations requested by telephone as verbal. Results: A total of 439 consultations requested in the ED were assessed for eligibility and 345 were included in the final analysis: 173 consultations were conducted using secure messaging application and 172 consultations were conducted using standard telephone communications. The median ED LOS was 240 minutes (IQR:230-270, 95\% Cl:240 to 255.2) for patients in the secure messaging application group and 277 minutes (IQR:270-287.8, 95\% Cl:277 to 279 ) for patients in the telephone group. The median total ED LOS was significantly lower among consults conducted using Secure messaging application relative to consults conducted by telephone (median dif: $-30,95 \% \mathrm{Cl}:-37$ to- 25 , $\mathrm{p}<0.0001$ ). The median consult time was 158 minutes (IQR:133 to $177.25,95 \%$ Cl:150 to 169 ) for patients in the Secure messaging application group and 170 minutes (IQR:165 to $188.5,95 \%$ Cl:170-171) for patients in the Telephone group (median dif: $-12,95 \% \mathrm{Cl}:-19$ to- $7, p<0.0001)$. Consultations completed without ED arrival was $61.8 \%$ in the secure messaging group and $33.1 \%$ in the Telephone group (dif: 28.7, $95 \% \mathrm{Cl}: 48.3$ to $66, \mathrm{p}<0.001$ ). Conclusions: Use of secure messaging application for consultations in the ED reduces the total ED LOS and consultation time. Consultation with secure messaging application eliminated more than half of in-person ED consultation visits.

\section{Correspondence to:}

Umut Gulacti, Assistant Prof.

Adiyaman University Medical Faculty Training and Research Hospital, Department of Emergency Medicine, Adiyaman/Turkey

Phone: +90(535) 5851900 Fax: +90(416) 2272753

Email: umutgulacti@gmail.com

\author{
Appl Clin Inform 2017; 8: 742-753 \\ received: April 18, 2017 \\ accepted in revised form: May 11, 2017 \\ published: July 19, 2017 \\ Gulacti U, Lok U. Comparison of secure messaging \\ application (WhatsApp) and standard telephone usage \\ for consultations on Length of Stay in the ED. Appl Clin \\ Inform 2017; 8: 742-753 \\ https://doi.org/10.4338/ACI-2017-04-RA-0064
}




\section{Background}

Emergency department (ED) crowding has been recognized as a serious concern and is associated with prolonged Emergency Department Length of Stay (ED LOS). Consultations occur frequently in the EDs (1). It is the process by which emergency physicians request other specialists (consultants) to participate in the care of the ED patients. Generally, as do most of the countries, the consultant should provide one of the following outcomes with this process: admission to hospital, discharge with or without consultant follow-up, completion of procedures or investigations, or consult another specialty in Turkey (2).

Hospitals in Turkey have different ED care systems due to type of hospital such as university hospital, affiliated hospital, training and research hospital and state hospital. Our hospital is affiliated with a state hospital. In our hospital, if primary care ED residents request consultations for patients, consultants (faculty or senior physicians) always return consultation requests. Residents from other departments such as surgery or internal medicine participate in ED patient care. Residents in the other departments can report to ED and take care of the patients.

Although many factors contribute to prolonged ED LOS, consultation difficulty and increased consultation time are the most important problems (1). In order to eliminate these problems, consultation methods including the use of telemedicine and SMS/MMS are beginning to be evaluated $(3,4)$. However, these methods have major disadvantages such as a prolonged consultation process for telemedicine methods and per message fees applied for the use of SMS/MMS (5-7). Therefore, healthcare providers and clinicians are increasingly exploring alternative communication technologies in order to facilitate and accelerate the consultation process (8). Smartphones are a common mode of communication in daily life. Almost all health professionals in the United States of America and the United Kingdom now carry smartphones (9). The widespread use of smartphones represents a new opportunity to integrate mobile technology into daily clinical practice. Despite concerns regarding security and privacy issues with certain applications, smartphones can have tremendous benefits in clinical practice (10). In addition, the development of new applications facilitates communication among users by improving the capture, storage, and transmission of images. The secure messaging application (WhatsApp Messenger) is a prominent example of this type of application (11). It, the most popular messaging application for smartphones worldwide, provides real time communication through text messages, videos, voice messages, and photographs. The secure messaging application has several advantages such as rapid transmission and no cost to the user (9). The secure messaging application has been used for clinical consultation, collaboration and communication between physicians in healthcare $(9,11-15,16)$.

The aim of this study was to evaluate the effect of secure messaging application usage for consultations on ED LOS and consult time.

\section{Methods}

\subsection{Study design and setting}

This prospective, randomized controlled clinical trial was conducted according to a detailed protocol that conforms to the CONSORT (Consolidated Standards of Reporting Trials) statement for reporting randomized trials. This study was planned as a superiority trial.

This study was conducted in the emergency department (ED) of a tertiary care university hospital (affiliated with state hospital) with an annual census of approximately 180,000 visits between 01, November 2015 and 01, February 2016. Consultations in this hospital are requested via telephone or secure messaging application. Consultants who are faculty or senior physician and residents from other departments are out of the hospital in many times in this hospital during night shifts. The pediatric ED is in a different location in the province. All diagnosis and treatment algorithms are standardized. All participants provided written informed consent.

The study was approved by the Medical Ethics Committee. Patient confidentiality was maintained by minimizing mention of patient identifiers. All precautions were taken to ensure that protocol of this study was compliant with the EU Data Protection Directive 95/46/EC which pro- 
tects individuals' privacy and personal data use. This study was registered to clinicaltrial.gov (Clinicaltrial ID of NCT02586779).

Consultations were randomized into two arms. The intervention group [secure messaging application group (WhatsApp)] included the consultations that were requested via the secure messaging application. The control group (Telephone group) included consultations that were requested by telephone as a verbal report.

\subsection{Selection of participants}

Adults (age $>18$ years) who were referred for consultation in the ED were eligible for the study. Participants were enrolled consecutively 24 hrs a day, 7 days a week. Study eligibility was determined by a resident working in the ED with attending physician. The consulted and consulting physicians who have a smartphone, who are familiar to use of secure messaging application, who are known as active secure messaging application user from previous communications and who have the connection to the internet network were enrolled in study. All consulted and consulting physicians were blinded to the purpose of the investigation.

Consultations, which was not determined real „consult time“ and the „ED LOS“, meeting any of the following criteria were excluded from the study: an unresponsive consultation (consultations not received response after two calls), an undetermined response time from the consulting physician, more than one consultation, re-admittance to the ED on the same day due to consultation failure, dead on arrival, left against medical advice, patients stayed in the ED after consultation.

\subsection{Randomization and interventions}

The randomization model was generated by a computer program. Opaque sealed envelopes were used to allocate the consultations requested into two groups. The randomization model and preparation of opaque sealed envelopes were performed by a person. The consultant specialist and the data collector were blinded to the purpose of the investigation.

The intervention group included consultations that were requested via secure messaging application. In intervention group, all communications between consultant and the ED physician were only generated via secure messaging application. A patient history, brief report and vital findings (blood pressure, heart and breathing rate, fever) by writing text messages ( Figure 1), and x-rays, ultrasound results, electrocardiograph, laboratory results, wound photographs, computerized tomography and magnetic resonance images, monitor rhythm sounds were sent to consultants as secure messaging messages. The control group included consultations that were requested by telephone. A brief patient history, blood pressure, heart and breathing rate, fever, Glasgow Coma Score, sensory-motor findings, neurovascular and movement examinations, and relevant investigations were transmitted to consultants as a verbal report. Eligible patients were enrolled in the study in a consecutive manner. After a patient was enrolled in the study, the times were noted by two medical secretaries, one of whom is responsible for record arrival time and one of whom is responsible for initiating consults and leave time from ED recorded as minutes to standardized study form.

\subsection{Outcome measurements}

Primary outcome measurement was the difference in total ED LOS between the two groups. Total LOS in the ED, was calculated as the ED arrival (hour and minutes when the patient first arrived) minus ED discharge (hour and minutes when the patient left the ED) (17) and was presented as minutes.

The secondary outcome measurement was the difference in consult time and termination of consultation between the two groups. Consult time was calculated as the first consultation time (time when the first consultation was requested) minus the time of disposition decision (time the bed was requested for admitted patients or time of ED discharge for discharged patients) (17) and was presented as minutes. In the time between the initiation and end of consultation, the ED physicians performed the advices of consultant (such as treatment, laboratory follow up, etc.). 


\subsection{Statistical analysis}

The numeric data were expressed by mean \pm standard deviation (SD) or median (interquartile range (IQR)) where applicable. Categorical data are expressed as rates. The Kolmogorov-Smirnov test was used to assess normality of continuous variables. The Mann-Whitney $U$ test was applied for the comparison of data that did not conform to a normal distribution. The study was designed as a superiority trial requiring 42 patients per group with an alpha critical value of $0.05,97 \%$ power, 12 minutes difference between groups and standard deviation of 15. All of the hypotheses were constructed as one tailed tests. A 95\% confidence interval (CI) was used for expressing the study data. The study data were analyzed using MedCalc Statistical Software for Windows, v12.7.0.0 (Ostend, Belgium) and $G^{*}$ power v3.1.9.2. For all analyses, statistical significance was defined as $\mathrm{p}<0.05$. All the analyses were performed according to the intention to treat analysis.

\section{Results}

We assessed 439 consecutive consultations for study eligibility. From the eligible consultations, 94 (21.4\%) consultations were excluded due to following reasons; 46 consultations (48.9\%) were more than one consultation, 18 (19.2\%) were stayed in the ED after consultation, 13 consultation (14.9\%) had undetermined response time, 11 patients $(11.7 \%)$ declined to participate $3(3.2 \%)$, patient had re-admittance to ED on the same day, 2 patients (2.1\%) left against medical advice, 1 patient (1.1\%) was dead on arrival. A total of 345 consultations were randomized for the study: 173 patients for the secure messaging group and 172 patients for the Telephone group. All the patients allocated to a study arm were evaluated by a consultant physician and included in the final analysis ( $>$ Figure 2). Consultant responded the consultation during second call for two patients in the intervention group and one patient in control group. However, these patients were included into the final analysis. The mean age of the study participants was $48.5 \pm 22.1$ years; $67.3 \%(n=231)$ of the study participants were men. There was no statistical difference was between two groups according to age, gender and numbers of consultation during night shifts ( $p>0.05$ for each other). The baseline characteristics are detailed in $>$ Table 1 .

Two hundred and seventy-three secure messaging application messages were sent to consultant specialists by Emergency physicians. Emergency physicians receipted from consultant specialists the 367 response messages as text message. Of 367 response messages, $58.0 \%(n=213)$ were instructiongiving comments, $30.2 \%(n=111)$ were the clinical questions, $\% 9.2(n=34)$ response messages were administrative questions and \%2.5 (n=9) were other questions. Any problem was not reported about secure messaging application by consulted physicians.

Median total ED LOS was 270 minutes (IQR:240 to 279) for all patients included in study. The median ED LOS was 240 minutes (IQR:230 to 270, 95\%CI: 240 to 255.2) for patients in the Secure messaging application group and 277 minutes (IQR:270 to $287.8,95 \% \mathrm{CI}: 277$ to 279 ) for patients in the Telephone group. The median total ED LOS of the Secure messaging application Group was significantly lower than the Telephone Group (median dif: $-30,95 \% \mathrm{CI}:-37$ to $-25, \mathrm{p}<0.0001$, $\triangleright$ figure 3a).

The median consult time was 170 minutes (IQR:150 to 180) for all patients included in the study. The median consult time was 158 minutes (IQR:133 to 177.25, 95\%CI:150 to169) for patients in the Secure messaging application group. The median consult time was 170 minutes (IQR:165 to 188.5, 95\% CI:170 to 171) for patients in Telephone group. The median total consult time of the secure messaging application Group was significantly lower than the Telephone Group (median dif.: -12, 95\% CI: -19 to $-7, \mathrm{p}<0.0001$, $>$ figure $3 \mathrm{~b}$ ).

Consultations completed without ED arrival was $61.8 \%(n=107)$ in the Secure messaging application group and $33.1 \%(\mathrm{n}=57)$ in the Telephone group (dif: 28.7, 95\% CI:48.3 to 66, $\mathrm{p}<0.001)$. Consultation with Secure messaging application eliminated more than half of in-person ED consultation visits. 


\section{Discussion}

Our findings suggest that the Secure messaging application reduces ED LOS and consult time for ED patients who require consultation. In traditional ED consultations, verbal report by telephone is the most commonly used method. This method does not allow for the transfer of X-rays, wound image, CT and MR images to the consulting physician (12).

Earlier studies have shown that consultation by telephone and/or other methods such as pager systems can result in a number of important limitations $(8,14,18,19)$. Tashkandy et al (21) found that consultation by telephone increased ED LOS.

To the best of our knowledge, this is the first randomized, double-blind clinical trial of the effect of secure messaging application usage on ED LOS and consultation time. The median total ED LOS was lower among consults conducted using secure messaging application relative to consults conducted by telephone. This may be explained by the view that the usage of secure messaging application lowers barrier to consultation and allows the resident to consult earlier, because more data can be shown via pictures and images to the consultant instead describing over the telephone.

Other studies have evaluated the effectiveness of secure messaging application implementation and use in health care. A study by Giordano et al (22) investigating consultations requested using the secure messaging application Messenger reported on the use of secure messaging application for initial documentation and transmission of images in the emergency department to more experienced consultants; the authors concluded that this approach is safe for clinical use. Another well-designed, prospective, study by Khanna et al (23) compared the effectiveness of communication prior to the introduction of secure messaging application and after the introduction of secure messaging application in an orthopedic clinic. They reported that the secure messaging application allows residents to function efficiently, improves communication and awareness regarding admitted patients, and facilitates handovers without significant disruptions to daily working routines.

A systematic review by Lee et al. (24) reported that consult time is the most time consuming procedure in the ED, and reducing consultation time may improve ED patient flow. A study by Samuel et al (25) found that the mean total consultation time for all consultants was greater than 2 hours. In our study, median consult time was 170 minutes for all consultations and consult time for consultations that were requested using the secure messaging application was shorter compared with consultations by Telephone.

Increasing the number of consultant visits to the ED leads to the increasing ED LOS and the ED crowding $(2,16,20,22)$. The most time consume process in consultation is the time arriving of consultant to the ED. A prospective cohort study by Brick et al. found that consult time was most influenced by time from arrival to consultation request. (26). In present study, the use of secure messaging application resulted in more consultations ending without the specialist attending the ED. A recent report by Qureshi et al (27) reported that consultations that required the presence of the consulting physician in the ED were the most time consuming. In the present study, $61.8 \%$ of consultations were completed using secure messaging application alone. Thus, consultation via secure messaging application reduced ED LOS by reducing the number of occasions on which a consultant needed to report to the ED.

Consulted physicians are able to look at radiographies in an electronic system in hospital. But, the consultant may not be able to connect to hospital database or may experience an inability in accessing patient information systems anywhere, if especially a clinician is mobile or out of hospital as do our country (2). However, secure messaging application is almost always with us. Therefore, consultants reach to all patient data anywhere.

The novelty of using app on the outcome is effect that advantage of secure messaging application send to consultant large amount of patients' data and consultant' decision is fasten and thus reduced to ED LOS. We thought that it may be preferred in patients who has not complex clinical condition and when specific radiographic images and laboratory data are ready at the time of consultation time. Thus, the consultation time reduction might get less.

There are some concerns about security and privacy issues associated with the use of communication technologies. However, until now, there is no scientific evidence that patient information which was sent to consultant via Secure messaging application reached the other person $(10,28)$ and this concerns is only a prediction. Secure messaging application has end-to-end encryption and 
when end-to-end encrypted, the messages, photos, videos, voice messages, documents, status updates and calls are secured from falling into the wrong hands. (16) Therefore, Secure messaging application is compatible with Health Insurance Portability and Accountability Act (HIPAA), which is United States legislation that provides data privacy and security provisions for safeguarding medical information (29). It ensures the protection of personal and contact data with a password. All necessary measures in communication via secure messaging application can be taken by the usage of password protection for both the smartphones and the application, screening and securing the devices with antivirus software along with prior instructions to the participating residents (23). Furthermore, to address concerns regarding the security of communications containing health data, health care providers or Health Ministry officials working in collaboration with information governance services may choose to create a new mobile application similar to secure messaging application program.

\section{Limitations}

This study has several limitations. First, despite high attendance number of our hospital, this study was conducted with small sample size because of study design. Patients' chronic illness, elapsed times for sending a secure messaging application message and ED LOS in patients requiring more than one consultation could not be evaluated. Second, how long it take physicians to do a consultation in telephone and Secure messaging application groups and consultation response times of consulted physicians were not compared between two groups. Third, there are a number of exclusions from this study, some of which may have had an impact on the results had they been included or are important outcomes when evaluating a new system for making referrals e.g. non responsive consultation, consultation failure. Because we could not calculate ED LOS and consult time of these consultations, we had to exclude these patients. A large multi-center study would help in the establishment role of secure messaging application on the ED LOS and consult time.

\section{Conclusion}

Duration of ED LOS and consult time for consultations requested via secure messaging application is shorter in comparison to conventional Telephone consultation. Consultations via Secure messaging application reduce the need for a consultant to report to the ED by more than half. The use of secure messaging application for consultations may significantly improve ED LOS and consult time.

ED crowding and prolonged ED LOS continues to be a major problem. Use of mobile Health applications has gained the momentum in recent years. This study demonstrates the effectiveness of simple messaging software to improve ED LOS and consult time. Further studies are needed for a more detailed description of secure messaging application use in the ED as new consultation method.

\section{Multiple Choice Questions}

What is benefit of using secure messaging application (WhatsApp) compared to telephone consultation?

A. Consult time is the shorter

B. ED LOS is the shorter

C. Reduce the need for a consultant to report to the ED by more than half

D. All of the above

Answer: D. Consult time and ED LOS is the shorter in consultations via secure messaging application than consultations via standard telephone and reduce the need for a consultant to report to the ED by more than half. 


\section{Clinical Relevance Statement}

Smartphone use, including healthcare applications, has increased among health care professional such as physicians. The widespread use of smartphones represents a new opportunity to integrate mobile technology into daily clinical practice. The secure messaging application, the most popular messaging application for smartphones, has been used for clinical consultation, collaboration and communication between physicians in clinical practice. Our study demonstrates that secure messaging application use in the ED as new medical consultation method is useful.

\section{Conflicts of Interest}

No conflict of interest was declared by the authors and the authors declared that this study has received no financial support.

\section{Human Subjects Protections}

The study was performed in compliance with the World Medical Association Declaration of Helsinki on Ethical Principles for Medical Research Involving Human Subjects and approved by the Medical Ethics Committee (date/number: 2015/04-11).

\section{Acknowledgement}

We would like to thank to Associate Professor Fatih Uckardes, Department of Biostatistics, Adiyaman University Medical Faculty, for the statistical revision of manuscript. 


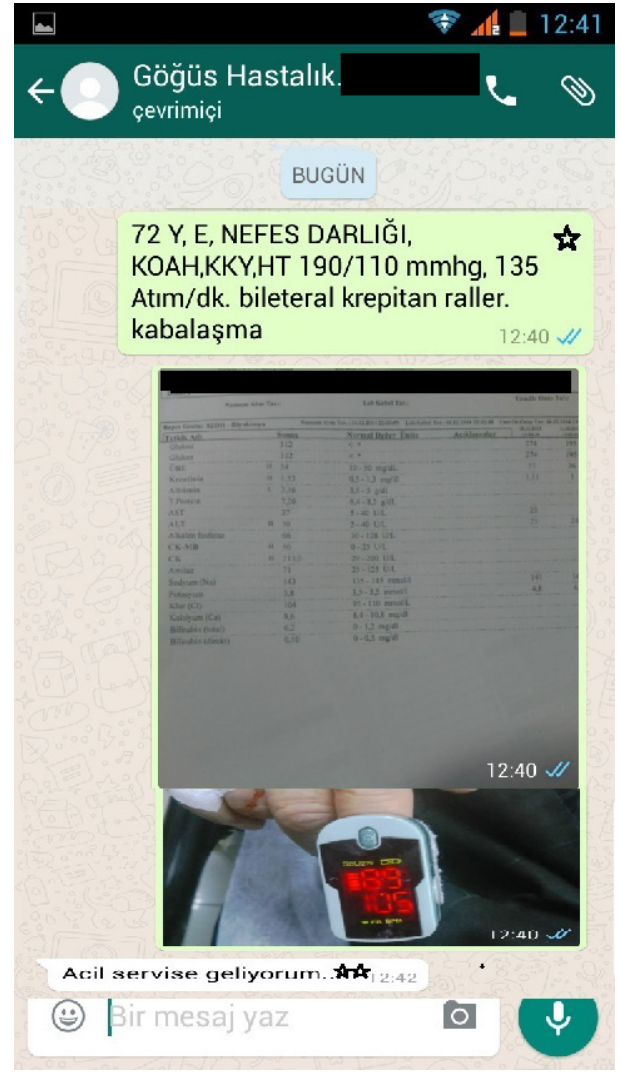

Fig. 1 Example of one of the communication via secure messaging application * (text message that emergency physician sent to consultant) (complaint): shortness of breath, COPD (chronic obstructive pulmonary disease), CHF (congestive heart failure), HT (hypertension), (TA): 190/70 mmHg, (Heart rate): $135 / \mathrm{min}$, Bilateral rales, ** (consultant' response) I'm coming to the ED.

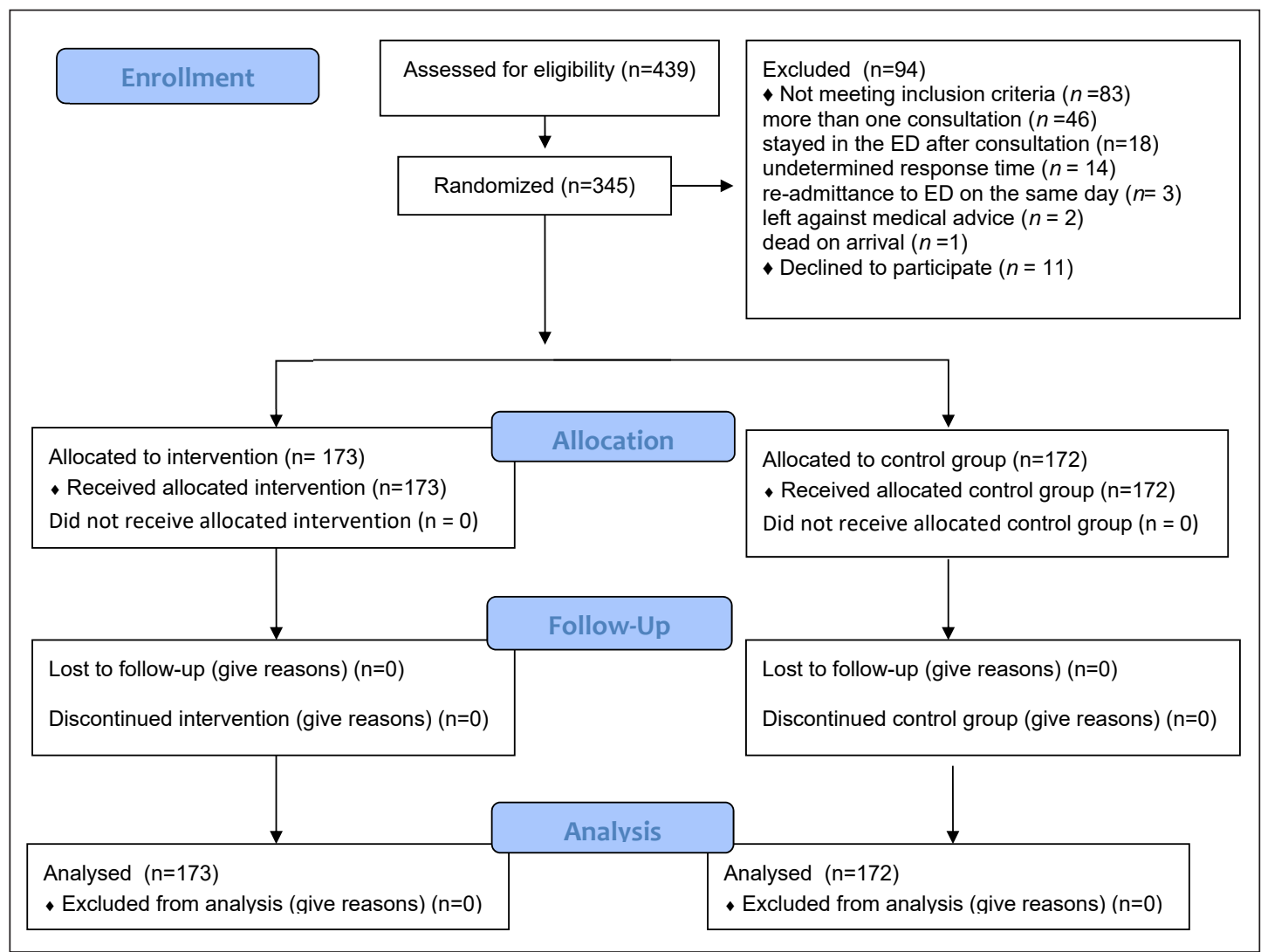

Fig. 2 CONSORT Patient flow diagram 


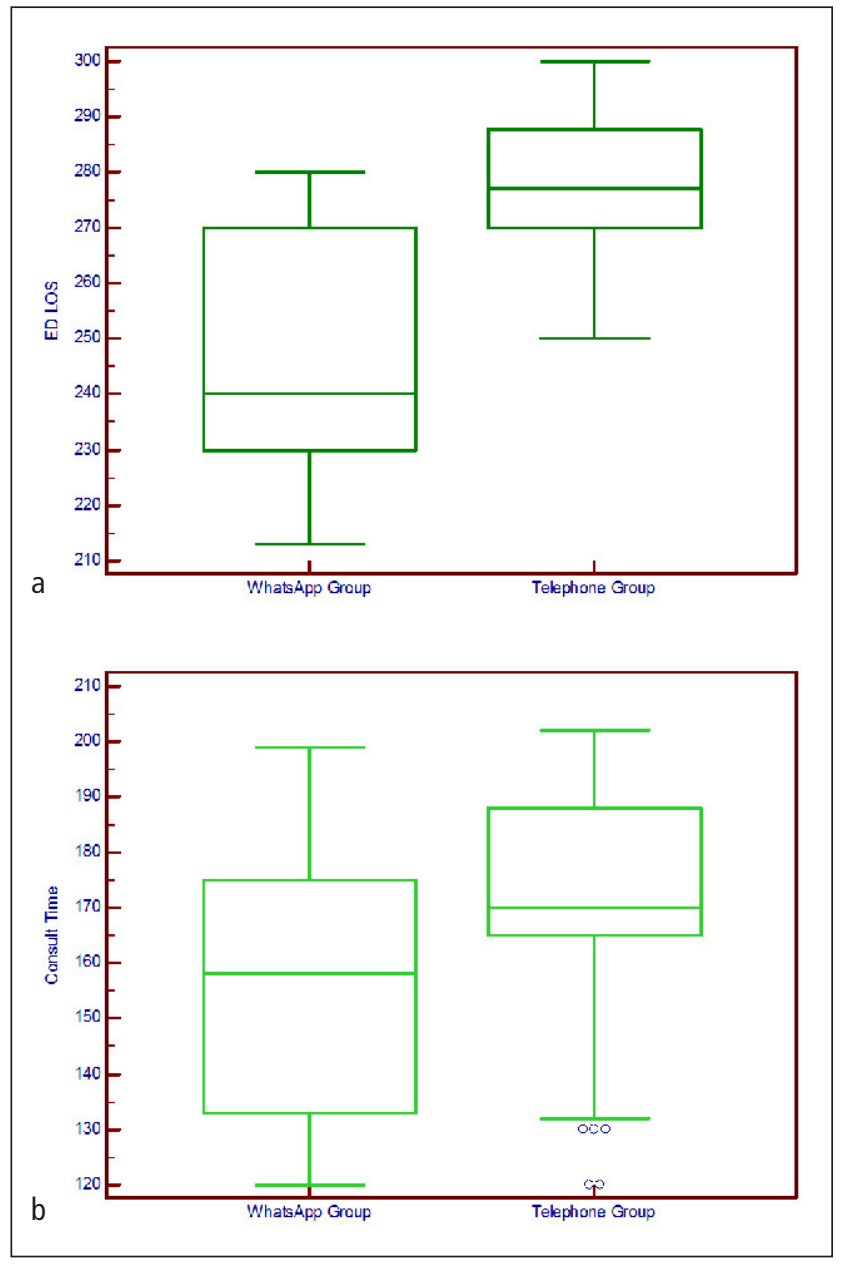

Fig. 3 Boxplot graphics of time differences between two groups; 3a. Difference of total Emergency Department Length of Stay between secure messaging application and Telephone Groups; 3b. Difference of Consult Time between Secure messaging application and Telephone Groups 
Table 1 Baseline characteristics of the requested consultations for secure messaging application and telephone groups

\begin{tabular}{|l|l|l|}
\hline Variables & WhatsApp Group (n=173) & Telephone Group (n=172) \\
\hline Age (years) \pm SD & $47.1 \pm 20.2$ & $46.5 \pm 22.9$ \\
\hline Male, no. (\%) & $111(64.2)$ & $120(70.6)$ \\
\hline $\begin{array}{l}\text { Consultation during night shifts, no. (\%) } \\
\text { Most common clinics requested consultation, no. (\%) }\end{array}$ & $96(55.8)$ \\
\hline Orthopaedics & $45(26.0)$ & $43(25.3)$ \\
\hline Cardiology & $44(25.4)$ & $42(24.4)$ \\
\hline Internal Medicine & $36(20.8)$ & $34(19.8)$
\end{tabular}

SD: Standard deviation 


\section{References}

1. Kang H, Nembhard HB, Rafferty C, DeFlitch CJ. Patient flow in the emergency department: a classification and analysis of admission process policies. Ann Emerg Med 2014; 64(4): 335-342.e8.

2. Erenler AK, Akbulut S, Güzel M, Çetinkaya H, Karaca A, Türköz B, Baydın A. Reasons of Overcrowding in The Emergency Department: Experiences And Suggestions of an Education And Research Hospital. Turk Journal of Emergency Medicine 2014; 14(2): 59-63

3. Agarwal AK, Gaieski DF, Perman SM, Abella BS, Carr BG. Emergency Department Based Telemedicine: Testing the Feasibility of Using Telemedicine as a Communication and Consultation Tool for Critically Ill Patients. Ann Emerg Med 2013; 62: 142-3

4. Kim M, Park J, Cho J, Chung S. Effect of a Short Message Service Program Regarding Patient Process Delays on Length of Stay in the Emergency Department. Ann Emerg Med 2011; 58: 293

5. Flodgren G, Rachas A, Farmer AJ, Inzitari M, Shepperd S.Interactive telemedicine: effects on professional practice and health care outcomes. Cochrane Database Syst Rev 2015; 9: CD002098.

6. Wong HT, Poon WS, Jacobs P, Goh KY, Leung CH, Lau FL, Kwok S, Ng S, Chow L. The comparative impact of video consultation on emergency neurosurgical referrals. Neurosurgery 2006; 59(3): 607-13; discussion 607-13.

7. Free C, Phillips G, Watson L, et al. The effectiveness of mobile health technologies to improve health care service delivery processes: a systematic review and meta-analysis. PLoS Med 2013; 10(1): e1001363.

8. Lo V, Wu RC, Morra D, Lee L, Reeves S. The use of smartphones in general and internal medicine units: a boon or a bane to the promotion of interprofessional collaboration?. J Interprof Care 2012; 26(4): 276-82.

9. Johnston MJ, King D, Arora S, Behar N, Athanasiou T, Sevdalis N, Darzi A. Smartphones let surgeons know Secure messaging application: an analysis of communication in emergency surgical teams. Am J Surg 2015; 209(1): 45-51.

10.Huffman A. With the Proliferation of Mobile Medical Apps, Which Ones Work Best in the Emergency Department? Ann Emerg Med 2015; 66: 14-5.

11. Astarcioglu MA, Sen T, Kilit C, Durmus HI, et al. Time-to-reperfusion in STEMI undergoing interhospital transfer using smartphone and WhatsApp messenger. Am J Emerg Med 2015; 33(10): 1382-4.

12. Wani SA, Rabah SM, Alfadil S, Dewanjee N, Najmi Y. Efficacy of communication amongst staff members at plastic and reconstructive surgery section using smartphone and mobile WhatsApp. Indian J Plast Surg 2013; 46(3): 502-5.

13. Petruzzi M, De Benedittis M. WhatsApp: a telemedicine platform for facilitating remote oral medicine consultation and improving clinical examinations. Oral Surg Oral Med Oral Pathol Oral Radiol 2016; 121(3): 248-54.

14.Dorwal P, Sachdev R, Gautam D, et al. Role of WhatsApp Messenger in the Laboratory Management System: A Boon to Communication. J Med Syst 2016; 40(1): 14.

15. Moy E, Coffey RM, Moore BJ, Barrett ML, Hall KK. Length of stay in EDs: variation across classifications of clinical condition and patient discharge disposition. Am J Emerg Med 2016; 34(1): 83-7.

16. Gulacti U, Lok U, Hatipoglu S, Polat H. An Analysis of WhatsApp Usage for Communication Between Consulting and Emergency Physicians. J Med Syst 2016; 40(6): 130.

17. Lee PA, Rowe BH, Innes G, Grafstein E, Vilneff R, Wang D, van Rheenen S, Lang E. Assessment of consultation impact on emergency department operations through novel metrics of responsiveness and decision-making efficiency. CJEM 2014; 16(3): 185-92.

18. Wu R, Rossos P, Quan S, et al. An evaluation of the use of smartphones to communicate between clinicians: a mixed-methods study. J Med Internet Res 2011; 13: e59.

19. Nguyen TC, Battat A, Longhurst C, et al. Alphanumeric paging in an academic hospital setting. Am J Surg 2006; 191: 561-5.

20. Coiera E, Tombs V. Communication behaviours in a hospital setting: an observational study. BMJ 1998; 316(7132): 673-6.

21. Tashkandy MA, Gazzaz ZJ, Farooq MU, Dhafar KO. Reasons for delay in inpatient admission at an emergency department. J Ayub Med Coll Abbottabad 2008; 20(1): 38-42.

22. Giordano V, Koch HA, Mendes CH, Bergamin A, de Souza FS, do Amaral NP. WhatsApp Messenger is useful and reproducible in the assessment of tibial plateau fractures: inter- and intra-observer agreement study. Int J Med Inform 2015; 84(2): 141-8.

23. Khanna V, Sambandam SN, Gul A, Mounasamy V. WhatsApp“ening in orthopedic care: a concise report from a 300-bedded tertiary care teaching center. Eur J Orthop Surg Traumatol 2015; 25(5): 821-6.

24.Lee RS, Woods R, Bullard M, Holroyd BR, Rowe BH. Consultations in the emergency department: a systematic review of the literature. Emerg Med J 2008; 25(1): 4-9. 
25. Samuel PA, Aldeen AZ, Gravenor S, Malik S. Analysis of Emergency Department Consultation Times. Annals of Emergency Medicine 2015; 66: 10.

26. Brick C, Lowes J, Lovstrom L, Kokotilo A, Villa-Roel C, Lee P, Lang E, Rowe BH. The impact of consultation on length of stay in tertiary care emergency departments. Emerg Med J 2014; 31(2): 134-8.

27. Qureshi NU, Ghawanni AO, Bakhider GO, AlDarrab A. Consultation Impacts on Patients' Length of Stay in the Emergency Department. Ann Emerg Med 2010; 56: 110-1.

28. Johnston M, King D, Darzi A. Reply to the letter: WhatsApp with patient data transmitted via instant messaging? Am J Surg. 2016; 211(1): 301-2.

29. www.hhs.gov [Internet]. Health Information Privacy. [cited 2017 May 05]. Available from: https://www. hhs.gov/hipaa/for-professionals/privacy/laws-regulations/. 